\title{
Defending subjective inflation: An inference to the best explanation
}

J.D. Knotts ${ }^{1}$, Matthias Michel $^{2,3}$, Brian Odegaard ${ }^{4}$

1. Department of Psychology, University of California, Los Angeles, USA

2. Centre for Philosophy of Natural and Social Science, London School of Economics and Political Science, London, UK.

3. Consciousness, Cognition \& Computation Group, Université Libre de Bruxelles, Belgium

4. Department of Psychology, University of Florida, Gainesville, USA.

\section{Corresponding Author:}

J.D. Knotts

502 Portola Plaza

Psychology Tower, Rm 8501

Los Angeles, CA 90095

jeffreydknotts@gmail.com

\begin{abstract}
In a recent opinion piece, (Abid 2019) criticizes the hypothesis that "subjective inflation" may partly account for apparent phenomenological richness across the visual field and outside the focus of attention (Knotts et al. 2018; Odegaard et al. 2018). In response, we address three main issues. First, we maintain that inflation should be interpreted as an intraperceptual - and not post-perceptual - phenomenon. Second, we describe how inflation may differ from "filling-in." Finally, we contend that, in general, there is sufficient evidence to tip the scales towards intraperceptual interpretations of visibility and confidence judgments.
\end{abstract}




\section{The central issues for inflation, and re-appealing to inference to the best explanation}

We previously argued that the debate about whether visual phenomenology is rich or sparse (Block 2011; Kouider et al. 2010; Lamme 2003, 2004; Phillips 2018; Ward 2018) may benefit from considering a psychological phenomenon referred to as "subjective inflation" (Odegaard et al., 2018; Knotts et al., 2018). Briefly, subjective inflation describes the finding that human observers exhibit liberal detection biases and overconfidence in their peripheral and/or unattended visual field, despite matched objective task performance for central and/or attended stimuli (Rahnev et al. 2011; Solovey, Graney, and Lau 2015; Odegaard et al. 2018; Li, Lau, and Odegaard 2018). We suggested that these findings may help explain the apparent richness we experience across the entire visual field, despite known physiological and behavioral deficits in the unattended periphery.

In a recent article, Abid (2019) points out that it is ambiguous whether the psychophysical findings supporting inflation are genuinely perceptual (or intraperceptual) as opposed to cognitive (or post-perceptual) in nature (Phillips 2011; Witt et al. 2015). While the inflation interpretation assumes that these effects are intraperceptual, Abid provides alternative post-perceptual interpretations, which he implies should be at least as compelling as their intraperceptual counterparts. Given these ambiguities, he suggests that we ought to remain agnostic about whether or not inflation effects have anything to do with phenomenology.

Abid also suggests that inflation posits an unfounded connection between decisional processes and phenomenology. According to him, this problem is "orthogonal" (p.3) to the problem of determining whether inflation is an intraperceptual or post-perceptual phenomenon. However, shortly after calling the two issues orthogonal, he shows that the former ultimately boils down to the latter:

If . . . there is some additional argument or evidence to be given in favor of the claim that there is a decisional component to perception, then it ought to be spelled out explicitly . . A Although the notion of a perceptual decision does play a central role in signal detection theory, the notion is ambiguous between a detection or discrimination process that is intraperceptual and one that is post-perceptual. (p. 4) 
Accordingly, despite his emphasis that the decisional issue is "precisely what is under dispute" (p. 5; see also his article's title), his arguments focus primarily on the issue of intra- versus post-perceptual ambiguity. Therefore, we focus on this issue here, which, we agree with Abid, is critical for inflation.

Since neither the intra- nor post-perceptual interpretation of a given behavioral effect can be directly decided by empirical evidence at the moment (Block 2007; Phillips 2011), we approach this debate about inflation effects as we have previously (Knotts et al. 2018), in the context of inference to the best explanation (Harman 1965; Block 2007). Specifically, we consider (1) whether there is sufficient evidence to tip the scales towards either the intra- or post-perceptual interpretation of relevant signal detection theory (SDT) criterion shifts, (2) whether, assuming the relevant criterion effects are intraperceptual, inflation is different from filling-in, and (3) whether there is sufficient evidence to tip the scales towards either the intra- or post-perceptual interpretation of visibility or confidence judgments.

\section{Are inflation effects measured by $c$ intra- or post-perceptual?}

Evidence supporting inflation includes the finding that observers tend to make higher numbers of false alarms in unattended or peripheral conditions in detection tasks that employ performance matching (Solovey, Graney, and Lau 2015; Rahnev et al. 2011). In SDT, this increase in false alarms manifests in the estimate of the perceptual criterion $(c)$ used to distinguish signal from noise (Macmillan and Douglas Creelman 2004; Green and Swets 1966). While changes in $c$ are associated with many perceptual illusions, such as the Müller-Lyer (Commons, Nevin, and Davison 1991; Witt et al. 2015), sound-induced flash (Shams, Kamitani, and Shimojo 2000; Rosenthal, Shimojo, and Shams 2009), and stream/bounce (Grove et al. 2012) illusions, Witt et al. (2015) have argued that such changes cannot distinguish intraperceptual from post-perceptual biases. This distinction is critical for the inflation hypothesis in that intraperceptual biases are assumed to reflect changes in phenomenology, whereas post-perceptual biases are not. 
One piece of evidence previously cited in favor of the intraperceptual interpretation of criterion effects is resistance to trial-by-trial feedback (Rosenthal, Shimojo, and Shams 2009; Rahnev et al. 2011; Solovey, Graney, and Lau 2015). The general intuition here is that if a bias is truly intraperceptual, there is no amount of explicit information to the contrary that can cause one to "unsee" it. In this sense, trial-by-trial feedback resistance is a sign of cognitive impenetrability, which is itself a criterion for determining whether a phenomenon is perceptual or post-perceptual (Firestone and Scholl 2016; Pylyshyn 1999). The Müller-Lyer illusion, which affects the criterion measure $c$, provides a good example (Witt et al. 2015). Even if one understands that a line with outward tails tends to appear longer than a nearby line of equal length with inward tails and modifies their responses accordingly, this knowledge and behavior do not eliminate the experience of the illusion (Brosvic and Finizio 1995).

In response, Abid argues that trial-by-trial feedback resistance is not necessarily evidence for the intra-perceptual interpretation because post-perceptual biases could also resist trial-by-trial feedback. This point is well taken. Perhaps we can benefit from inference to the best explanation. What might a post-perceptual feedback-resistant bias look like? And, does it seem more or less plausible than the intraperceptual alternative?

Abid provides examples; he cites "racist, religious, and superstitious biases" (p. 3) as being putatively both post-perceptual and feedback-resistant. However, do we expect such high-level, social and spiritual biases to show up in the types of controlled, low-level psychophysics experiments supporting the inflation hypothesis? We believe it is reasonable to assume that the answer to this question is no. One of the virtues of psychophysics is that we do not have to worry about such personal-level biases. If there are better, lower-level examples of post-perceptual feedback resistant biases, Abid does not provide them. Thus, on this example 
alone, we argue that inference to the best explanation should point at least marginally toward the intraperceptual interpretation.

Debates on how to separate intra- and post-perceptual effects are not new. For instance, multisensory research has engaged similar issues: when perceptual reports for one sensory modality are modified by sensory input in another, is it due to post-perceptual bias, or a true change in phenomenology (Choe et al. 1975)? The field has largely settled on the intraperceptual interpretation by (1) trusting that subjective reports index phenomenology (Bertelson and Radeau 1976), (2) demonstrating feedback resistance (Rosenthal, Shimojo, and Shams 2009), and (3) anatomical evidence supporting multimodal interactions in early levels of the sensory hierarchy (Kayser et al. 2005; Kayser, Petkov, and Logothetis 2009; M. M. Murray et al. 2016; Ghazanfar and Schroeder 2006). We have made the case for the first two criteria in inflation.

Regarding the third criterion, while much work on the neural basis of inflation remains to be done, several sources of evidence indicate that criterion effects - such as those observed in inflation - can be intraperceptual. For instance, (Jin and Glickfeld 2019) have used optogenetic manipulation of the excitability of V1 to change early sensory encoding in a detection task, and induce liberal (or conservative) criteria without changing sensitivity $(d)$. As another example, high-prestimulus neural excitability - indexed by $\alpha$ and $\beta$ power - can induce a liberal bias in detection tasks, as well as higher visibility and confidence ratings, while leaving sensitivity in discrimination tasks unchanged (Samaha, Iemi, and Postle 2017; Samaha, LaRocque, and Postle 2020; Benwell et al. 2017). A study by Iemi \& Busch (2018), which leverages opposing interpretations of criterion bias in two-interval forced choice detection and discrimination tasks, respectively, suggests that these prestimulus excitability effects on the criterion are intraperceptual. And further studies have indicated that these effects likely operate by modulating sensory evidence accumulation in the visual cortex (Kloosterman et al. 2019; Samaha, LaRocque, and Postle 2020; Samaha et al. 2020). 
Finally, expectation-based detection and discrimination biases - such as those we have argued for with respect to inflation (Knotts et al. 2018) - are associated with processing in early visual cortex (Kok et al. 2013; Pajani et al. 2015; Mostert, Kok, and de Lange 2015) and in areas as low-level as the superior colliculus (Crapse, Lau, and Basso 2018). While the neural locus of inflation need not necessarily be constrained to early sensory regions, these findings provide additional evidence that the biases underlying inflation could be intraperceptual in nature.

\section{If the relevant changes in $c$ are intraperceptual, is inflation distinct from filling-in?}

Another criticism from Abid is the following: if the relevant changes in $c$ are intraperceptual, then inflation is not distinct from the phenomenon of perceptual "filling-in" (Komatsu 2006). However, if these changes in $c$ are post-perceptual, then inflation is irrelevant for phenomenology. Regardless of which option is correct, inflation fails to contribute anything meaningful to the debate.

Reiterating the operational definition of inflation may help clarify what it contributes to the debate about phenomenology. Inflation occurs when there is a difference in subjective ratings between two conditions for which objective performance is matched. On this definition alone, inflation is, at least in theory, distinct from filling-in; one can imagine there being filled-in perceptual content for which subjective judgments are not inflated. And one can imagine there being inflated subjective judgments that are not based on filled-in perceptual content. However, giving Abid the benefit of the doubt, we might hypothesize that these two phenomena always co-occur, rendering them, in practice, indistinct. If this is true, then wherever filling-in is observed, inflated subjective judgments should be observed as well. 
Some preliminary evidence for filled-in representations being subjectively inflated comes from an elegant study by Ehinger et al. (2017), in which participants were more likely to choose a filled-in striped pattern than a veridical one when forced to indicate which of the two patterns was continuously striped. Similar evidence has been found for filled-in foveal content under low light conditions (Gloriani and Schütz 2019), but it remains an open question whether inflated subjective judgments are an intrinsic feature of filling-in, or, rather, if inflation is an independent, dissociable process. The same question can be asked of the potential relationship between subjective inflation and other peripheral mechanisms that involve summary statistics (Cohen, Dennett, and Kanwisher 2016). For example, are subjective ratings for crowded stimuli inherently inflated, as has been observed in at least one case (Odegaard et al. 2018)?

These questions are empirically tractable, and should be investigated in future studies. But, regardless of whether inflation is ultimately an inherent feature of other known peripheral summary mechanisms or an independent, dissociable process, what is important is that it provides explanatory power for a fundamental question about the apparent richness of phenomenology: why, if peripheral vision is coarse and summarized, does it not feel coarse and summarized? It feels so because subjective evaluation of these coarse and summarized representations is systematically biased, or inflated, relative to what we would predict based on the relationship between objective and subjective judgments in typical, foveal vision. This empirically tractable operationalization is the "novel evidence aimed at explaining the apparent richness of visual phenomenology" (Abid, 2019, p. 4) that the inflation hypothesis offers.

While operational definitions are useful for understanding inflation from a behavioral standpoint, we agree with Abid that more precisely characterizing "what it is like" to have inflated phenomenology, and how it may differ from "what it is like" to experience filled-in content, is essential. The phenomenological difference between modal and amodal completion may provide a helpful analogy. Modal completion involves the 
subjective visual perception of filled-in contours and surfaces. For example, one can clearly see filled-in, illusory contours between the 'Pac-Men'-like shapes at each corner of a Kanizsa square (Figure 1A). In modal completion, completion operates by a form of filling-in that has modal features (e.g. visual features). In amodal completion, on the other hand, the completed parts are not subjectively visualized - which is why it is amodal - but are still visually represented, giving rise to a visual sense of presence of occluded figures (Figure 1B; Michotte and Burke 1951; Thielen et al. 2019; van Lier and Gerbino 2015).
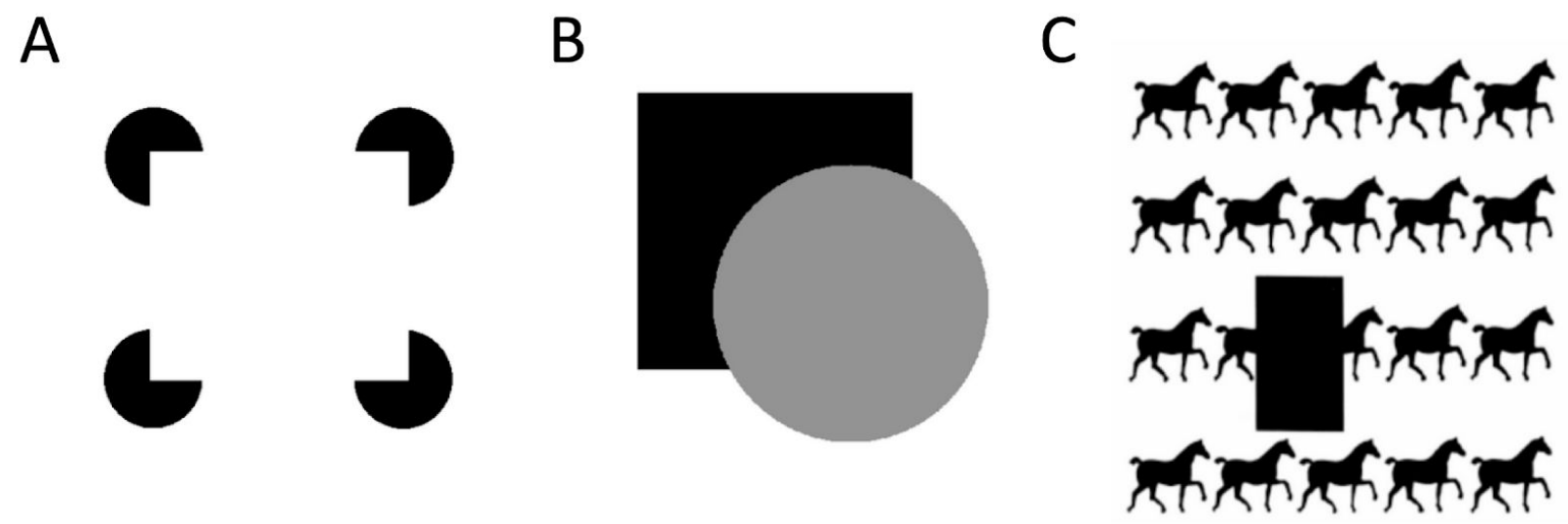

Figure 1. (A) Kanizsa square: an example of modal completion. (B) The partly occluded square is amodally completed, and thus seems perceptually present behind the occluding disk. (C) Amodal completion results in the feeling of presence of a long horse behind the rectangle occluder, instead of two horses. As noted by (Kanizsa 1985), these and similar examples suggest that amodal completion is partly independent from logical reasoning and expectations, thereby indicating that it is not a purely post-perceptual phenomenon (Nanay 2010).

Importantly, evidence suggests that amodal completion is at least partly a perceptual process: it can be cognitively impenetrable (Ekroll, Sayim, and Wagemans 2017; Ekroll et al. 2016; Kanizsa 1985; Figure 1C); it occurs relatively early on in visual processing (Sekuler and Palmer 1992; Chen et al. 2009; R. F. Murray, Sekuler, and Bennett 2001); occluded objects pop-out as do their unoccluded counterparts in visual search (Rensink and Enns 1998); and amodally completed objects can induce adaptation effects that are similar to those induced by their unoccluded counterparts (Fang and He 2005; Weisstein, Montalvo, and Ozog 1972). 
Although amodal completion and subjective inflation probably depend on different neurocognitive mechanisms, we suggest that, at the phenomenological level, perceptual inflation may feel somewhat like amodal completion. Both phenomena involve a perceptual sense of presence that is distinct from active filling-in, or conscious perception of qualitatively visual features, such as colors. In that sense, one can phenomenologically characterize subjective inflation in the visual periphery as a feeling of presence of visual features, similar to the feeling of presence elicited by looking at the square in Figure 1B, or the "sausage" horse in Figure 1C. In much the same way that amodal completion is thought to be a perceptual phenomenon, and is different from modal completion, we hold that subjective inflation is a perceptual phenomenon, distinct from filling-in.

Of course, this does not mean that filling-in does not contribute to the overall sense of phenomenal richness. Presumably, the two processes would be complementary (e.g., as we have described for the studies by Gloriani and Schütz (2019) and Ehinger et al. (2017)). Further, the distinction between modal and amodal completion does not, in and of itself, provide support for the idea that filling-in and inflation are, in practice, dissociable processes. As mentioned above, it may be that filled-in representations always display the operational signature of inflation. The analogy to modal and amodal completion is simply intended to provide a more intuitive sense of how the two processes could differ in terms of their respective contributions to phenomenology.

\section{Are subjective judgments intra- or post-perceptual?}

Abid's last major criticism of inflation regards the validity of subjective judgments as measures of phenomenology. His primary challenge is that sources of information other than introspection about visual phenomenology may contaminate subjective judgments. This is analogous to his argument about post-perceptual interpretations of SDT criterion shifts. For example, regarding confidence judgments, he states that "A student deep in the thick of Cartesian skepticism may lack confidence in all their perceptual 
judgments, but it would be preposterous to claim that reading Descartes might render one visually impaired" (p.4).

We agree and suggest that precisely what makes such a claim - which amounts to an odd type of Orwellian postdiction (Phillips, 2011) - preposterous is that Abid is describing a different type of confidence judgment than what is typically asked for in perceptual tasks. One amounts to an abstract, philosophical position, while the other is a simple metacognitive judgment of task performance. If a Cartesian skeptic with a normally functioning visual system repeatedly rates "zero confidence" in their performance on a task that they are performing significantly above chance, then the simplest explanation is that they are failing to comply with task instructions. But we do not need to worry about such cases as long as we do our due diligence as experimenters and provide clear instructions. If, on the other hand, Abid suggests that study participants should not be trusted to follow task instructions in general, then his argument overgeneralizes to psychological research as a whole.

Abid provides other examples of a similar flavor. For instance, he suggests that the overconfidence observed in crowding (Odegaard et al. 2018) may be due to participants' knowledge "that they can often discriminate objects surrounded by other objects in non-laboratory settings: they simply need to move their eyes and fixate them." While we cannot definitively rule out this possibility, is it the best explanation of the observed phenomenon? This interpretation fails to acknowledge that overconfidence was observed for crowded stimuli relative to non-crowded stimuli. Why would the knowledge that eye movements can improve perceptual inference apply more to the crowded condition? Again, Abid offers a tenable but uncompelling example of a post-perceptual bias that could contaminate an intended measure of phenomenology. Inference to the best explanation again favors the simpler interpretation that participant's confidence judgments meaningfully reflect what they see. 
While Abid explicitly targets studies using visibility (Rahnev et al. 2011) and confidence ratings (Odegaard et al. 2018) cited as evidence in support of subjective inflation, his arguments generalize to all studies that use confidence and visibility judgments as measures of phenomenology, by groups on all sides of many key debates in consciousness science (e.g., Landman, Spekreijse, and Lamme 2003; Ramsøy and Overgaard 2004; Dehaene and Naccache 2001; King and Dehaene 2014; Bronfman et al. 2014; Maniscalco and Lau 2016; Webb et al. 2016; Matthews et al. 2018; Ward, Bear, and Scholl 2016). Ultimately, any result in the psychology of perception relying on any kind of reports can be re-interpreted as post-perceptual, provided that one is willing to assume the existence of sufficiently complex post-perceptual biases. However, it would be incorrect to conclude, for instance, that visibility judgments never tell us anything about phenomenology simply because alternative post-perceptual explanations of these judgments are conceivable, or because those judgments can sometimes be dissociated from phenomenology ${ }^{1}$. To avoid this overgeneralization, we suggest that subjective judgments should be considered meaningful indicators of phenomenology until proven guilty of post-perceptual influences.

But this is not to say that subjective measures are simply "better than nothing." In addition to their face validity for assessing phenomenology, recent work explains the utility and relevance of using confidence ratings in paradigms where performance is matched to probe conscious awareness of phenomenological content (Morales, Odegaard, and Maniscalco 2019), and at least some perceptual findings using confidence and visibility judgments cannot be accounted for by post-decisional cognitive/memory biases (Samaha and Denison 2020). Researchers can also analyze visibility and confidence judgments within the framework of

\footnotetext{
${ }^{1}$ Finding a double dissociation between a measurement indicator $I$ and a phenomenon $P$ does not imply that, in general, $I$ is not a good indicator of $P$. For instance, in the general population height is a good indicator of weight, even if double dissociations are obviously possible. In the same way, sensations of hot and cold can be doubly dissociated from temperature, but it would be absurd to conclude that sensations of hot and cold are never good indicators of temperature (Chang 2004). So, the fact that confidence and visibility judgments can sometimes be dissociated from phenomenology is not good evidence against their prima facie reliability in most cases.
} 
SDT (Galvin et al. 2003; Maniscalco and Lau 2012), which offers rigorous tools for quantifying the effects of biases in experimental settings. This type of empirical rigor stands in contrast to recent appeals to use open-ended picture drawing and verbal descriptions to probe phenomenology (Haun et al. 2017). While such approaches are not necessarily without merit, they are just as susceptible to post-perceptual re-interpretation, while lacking the benefits of fitting within a quantitative framework. Thus, if the goal is to have a true science of conscious awareness that involves testable predictions and quantifiable measures, confidence and visibility judgments currently represent our best option, as evidenced by their pervasive use in consciousness research. Of course, this is not to say that they represent a perfect, linear index of phenomenology, as we have acknowledged previously (Odegaard et al. 2018; Knotts et al. 2018; Michel 2019).

\section{Conclusion \& Future Directions}

Overall, we appreciate Abid's appeal to skepticism given the current limits on what we can unequivocally infer about consciousness from empirical investigation (Block 2007; Phillips 2011). However, we agree with Block (2007) that given this difficulty, progress in our understanding of consciousness hinges critically on inference to the best explanation. In this regard, we have argued that shifts in both the SDT criterion and subjective judgments that have been cited as evidence for subjective inflation (Odegaard et al. 2018; Knotts et al. 2018) are best explained as being intraperceptual. We have also attempted to clarify, by way of analogy to amodal completion, the putative qualitative nature of inflated phenomenology.

There are still many questions about inflation that need answering. For example, why does inflation occur? Despite Abid's puzzling claim to the contrary (Abid, 2019, p. 6), we have already written on this issue

(Odegaard et al. 2018; Knotts et al. 2018), suggesting that it may be linked to self-consistent perception

(Stocker and Simoncelli 2006; Luu and Stocker 2016), or could be due to reliance on priors (Kouider and Dupoux 2004; Kouider, De gardelle, and Dupoux 2007) and/or summary representations (Cohen, Dennett, 
and Kanwisher 2016) when information is sparse. However, arguments can only take this debate so far in the near future. What we need is more data to test whether or not this phenomenon extends beyond what has been shown thus far, including the degree to which it does (or does not) extend across different perceptual domains, the degree to which it is (or is not) supported by paradigms requiring different degrees of decisional complexity (detection vs. discrimination vs. identification; Odegaard and Lau 2016), the degree to which it does (or does not) operationally dissociate from filling-in, and the degree to which it may be compatible (or not) with contemporary ideas and theories about why we have phenomenology at all (Gershman 2019; Lau 2019). The full explanatory power of inflation can be explored in tests that we have outlined previously (Knotts et al. 2018; Odegaard et al. 2018), and we look forward to seeing whether this idea can continue to provide insight regarding a historically difficult scientific problem.

\section{Acknowledgements}

The authors thank Hakwan Lau for helpful feedback on the manuscript. MM was supported by the Fondation ULB.

\section{Data Availability}

There is no specific data to make available with this article.

\section{References}

Abid, Greyson. 2019. "Deflating Inflation: The Connection (or Lack Thereof) between Decisional and Metacognitive Processes and Visual Phenomenology.” Neuroscience of Consciousness 2019 (1): niz015.

Benwell, Christopher S. Y., Chiara F. Tagliabue, Domenica Veniero, Roberto Cecere, Silvia Savazzi, and Gregor Thut. 2017. "Prestimulus EEG Power Predicts Conscious Awareness But Not Objective Visual Performance." eNeuro 4 (6). https://doi.org/10.1523/ENEURO.0182-17.2017.

Bertelson, Paul, and Monique Radeau. 1976. "Ventriloquism, Sensory Interaction, and Response Bias: Remarks on the Paper by Choe, Welch, Gilford, and Juola." Perception \& Psychophysics 19 (6): 531-35.

Block, Ned. 2007. "Consciousness, Accessibility, and the Mesh between Psychology and Neuroscience." The Behavioral and Brain Sciences 30 (5-6): 481-99; discussion 499-548.

- 2011. "Perceptual Consciousness Overflows Cognitive Access." Trends in Cognitive Sciences 15 (12): $567-75$. 
Bronfman, Zohar Z., Noam Brezis, Hilla Jacobson, and Marius Usher. 2014. "We See More Than We Can Report: 'Cost Free' Color Phenomenality Outside Focal Attention.” Psychological Science 25 (7): 1394-1403.

Brosvic, G. M., and S. Finizio. 1995. "Inaccurate Feedback and Performance on the Müller-Lyer Illusion." Perceptual and Motor Skills 80 (3): 896-98.

Chang, Hasok. 2004. Inventing Temperature: Measurement and Scientific Progress. Oxford University Press.

Chen, Juan, Bingyun Liu, Bing Chen, and Fang Fang. 2009. "Time Course of Amodal Completion in Face Perception." Vision Research 49 (7): 752-58.

Choe, Chong S., Robert B. Welch, Robb M. Gilford, and James F. Juola. 1975. “The 'ventriloquist Effect': Visual Dominance or Response Bias?” Perception \& Psychophysics 18 (1): 55-60.

Cohen, Michael A., Daniel C. Dennett, and Nancy Kanwisher. 2016. "What Is the Bandwidth of Perceptual Experience?” Trends in Cognitive Sciences 20 (5): 324-35.

Commons, Michael L., John A. Nevin, and Michael C. Davison, eds. 1991. "Signal Detection: Mechanisms, Models, and Applications." Quantitative Analyses of Behavior Series. 285. https://psycnet.apa.org/fulltext/1991-97845-000.pdf.

Crapse, Trinity B., Hakwan Lau, and Michele A. Basso. 2018. "A Role for the Superior Colliculus in Decision Criteria." Neuron 97 (1): 181-94.e6.

Dehaene, S., and L. Naccache. 2001. "Towards a Cognitive Neuroscience of Consciousness: Basic Evidence and a Workspace Framework." Cognition 79 (1-2): 1-37.

Ehinger, Benedikt V., Katja Häusser, José P. Ossandón, and Peter König. 2017. "Humans Treat Unreliable Filled-in Percepts as More Real than Veridical Ones.” eLife 6 (May): e21761.

Ekroll, Vebjørn, Bilge Sayim, Ruth Van der Hallen, and Johan Wagemans. 2016. "Illusory Visual Completion of an Object's Invisible Backside Can Make Your Finger Feel Shorter." Current Biology: CB 26 (8): 1029-33.

Ekroll, Vebjørn, Bilge Sayim, and Johan Wagemans. 2017. “The Other Side of Magic.” Perspectives on Psychological Science: A Journal of the Association for Psychological Science 12 (1): 91-106.

Fang, Fang, and Sheng He. 2005. "Viewer-Centered Object Representation in the Human Visual System Revealed by Viewpoint Aftereffects." Neuron 45 (5): 793-800.

Firestone, Chaz, and Brian J. Scholl. 2016. "Cognition Does Not Affect Perception: Evaluating the Evidence for 'Top-down' Effects.” The Behavioral and Brain Sciences 39 (January): e229.

Galvin, Susan J., John V. Podd, Vit Drga, and John Whitmore. 2003. "Type 2 Tasks in the Theory of Signal Detectability: Discrimination between Correct and Incorrect Decisions." Psychonomic Bulletin \& Review 10 (4): 843-76.

Gershman, Samuel J. 2019. "The Generative Adversarial Brain." Frontiers in Artificial Intelligence and Applications 2: 18.

Ghazanfar, Asif A., and Charles E. Schroeder. 2006. "Is Neocortex Essentially Multisensory?" Trends in Cognitive Sciences 10 (6): 278-85.

Gloriani, Alejandro H., and Alexander C. Schütz. 2019. "Humans Trust Central Vision More Than Peripheral Vision Even in the Dark." Current Biology: CB 29 (7): 1206-10.e4.

Green, David M., and John A. Swets. 1966. Signal Detection Theory and Psychophysics. New York, NY: Wiley.

Grove, Philip M., Jessica Ashton, Yousuke Kawachi, and Kenzo Sakurai. 2012. “Auditory Transients Do Not Affect Visual Sensitivity in Discriminating between Objective Streaming and Bouncing Events." Journal of Vision 12 (8): 5.

Harman, Gilbert H. 1965. "The Inference to the Best Explanation.” The Philosophical Review 74 (1): 88-95.

Haun, Andrew M., Giulio Tononi, Christof Koch, and Naotsugu Tsuchiya. 2017. "Are We Underestimating the Richness of Visual Experience?” Neuroscience of Consciousness 2017 (1). https://doi.org/10.1093/nc/niw023.

Iemi, Luca, and Niko A. Busch. 2018. "Moment-to-Moment Fluctuations in Neuronal Excitability Bias 
Subjective Perception rather than Strategic Decision-Making.” eNeuro 5 (3): 1-13.

Jin, Miaomiao, and Lindsey L. Glickfeld. 2019. "Contribution of Sensory Encoding to Measured Bias." The Journal of Neuroscience 39 (26): 5115-27.

Kanizsa, G. 1985. "Seeing and Thinking." Acta Psychologica 59 (1): 23-33.

Kayser, Christoph, Christopher I. Petkov, Mark Augath, and Nikos K. Logothetis. 2005. "Integration of

Touch and Sound in Auditory Cortex." Neuron 48 (2): 373-84.

Kayser, Christoph, Christopher I. Petkov, and Nikos K. Logothetis. 2009. "Multisensory Interactions in

Primate Auditory Cortex: fMRI and Electrophysiology.” Hearing Research 258 (1-2): 80-88.

King, J-R, and S. Dehaene. 2014. "A Model of Subjective Report and Objective Discrimination as Categorical Decisions in a Vast Representational Space." Philosophical Transactions of the Royal Society of London. Series B, Biological Sciences 369 (1641). https://doi.org/10.1098/rstb.2013.0204.

Kloosterman, Niels A., Jan Willem de Gee, Markus Werkle-Bergner, Ulman Lindenberger, Douglas D. Garrett, and Johannes Jacobus Fahrenfort. 2019. "Humans Strategically Shift Decision Bias by Flexibly Adjusting Sensory Evidence Accumulation.” eLife 8 (February): e37321.

Knotts, J. D., Brian Odegaard, Hakwan Lau, and David Rosenthal. 2018. “Subjective Inflation: Phenomenology's Get-Rich-Quick Scheme.” Current Opinion in Psychology 29 (November): 49-55.

Kok, Peter, Gijs Joost Brouwer, Marcel A. J. van Gerven, and Floris P. de Lange. 2013. "Prior Expectations Bias Sensory Representations in Visual Cortex." The Journal of Neuroscience: The Official Journal of the Society for Neuroscience 33 (41): 16275-84.

Komatsu, Hidehiko. 2006. "The Neural Mechanisms of Perceptual Filling-In.” Nature Reviews. Neuroscience 7 (3): $220-31$.

Kouider, Sid, Vincent De gardelle, and Emmanuel Dupoux. 2007. "Partial Awareness and the Illusion of Phenomenal Consciousness." The Behavioral and Brain Sciences 30 (5-6): 510-11.

Kouider, Sid, and Emmanuel Dupoux. 2004. "Partial Awareness Creates the 'Illusion' of Subliminal Semantic Priming." Psychological Science 15 (2): 75-81.

Kouider, Sid, Vincent de Gardelle, Jérôme Sackur, and Emmanuel Dupoux. 2010. "How Rich Is Consciousness? The Partial Awareness Hypothesis." Trends in Cognitive Sciences 14 (7): 301-7.

Lamme, Victor. 2003. "Why Visual Attention and Awareness Are Different." Trends in Cognitive Sciences 7 (1): $12-18$.

- 2004. "Separate Neural Definitions of Visual Consciousness and Visual Attention; a Case for Phenomenal Awareness." Neural Networks 17 (5-6): 861-72.

Landman, Rogier, Henk Spekreijse, and Victor Lamme. 2003. "Large Capacity Storage of Integrated Objects before Change Blindness." Vision Research 43 (2): 149-64.

Lau, Hakwan. 2019. "Consciousness, Metacognition, \& Perceptual Reality Monitoring." Psy ArXiv. https://doi.org/10.31234/osf.io/ckbyf.

Lier, Rob van, and Walter Gerbino. 2015. "Perceptual Completions." In The Oxford Handbook of Perceptual Organization, edited by Johan Wagemans. Oxford University Press.

Li, Musen Kingsley, Hakwan Lau, and Brian Odegaard. 2018. "An Investigation of Detection Biases in the Unattended Periphery during Simulated Driving." Attention, Perception \& Psychophysics 80 (6): 1325-32.

Luu, Long, and Alan A. Stocker. 2016. "Choice-Induced Biases in Perception." bioRxiv. https://doi.org/10.1101/043224.

Macmillan, Neil A., and C. Douglas Creelman. 2004. Detection Theory: A User's Guide. Psychology Press. Maniscalco, Brian, and Hakwan Lau. 2012. "A Signal Detection Theoretic Approach for Estimating Metacognitive Sensitivity from Confidence Ratings.” Consciousness and Cognition 21 (1): 422-30.

- 2016. "The Signal Processing Architecture Underlying Subjective Reports of Sensory Awareness." Neuroscience of Consciousness 2016 (1). https://doi.org/10.1093/nc/niw002.

Matthews, Julian, Pia Schröder, Lisandro Kaunitz, Jeroen J. A. van Boxtel, and Naotsugu Tsuchiya. 2018. "Conscious Access in the near Absence of Attention: Critical Extensions on the Dual-Task Paradigm." 
Philosophical Transactions of the Royal Society of London. Series B, Biological Sciences 373 (1755): 20170352.

Michel, Matthias. 2019. "The Mismeasure of Consciousness: A Problem of Coordination for the Perceptual Awareness Scale.” Philosophy of Science 86 (5): 1239-49.

Michotte, A., and L. Burke. 1951. "Une Nouvelle Enigme Dans La Psychologie de La Perception: Le'donne Amodal'dans L'experience Sensorielle." Proceedings and Papers of the 13th International Congress of Psychology, 179-80.

Morales, Jorge, Brian Odegaard, and Brian Maniscalco. 2019. "The Neural Substrates of Conscious

Perception without Performance Confounds.” https://doi.org/10.31234/osf.io/8zhy3.

Mostert, Pim, Peter Kok, and Floris P. de Lange. 2015. "Dissociating Sensory from Decision Processes in Human Perceptual Decision Making." Scientific Reports 5 (December): 18253.

Murray, Micah M., Antonia Thelen, Gregor Thut, Vincenzo Romei, Roberto Martuzzi, and Pawel J. Matusz. 2016. “The Multisensory Function of the Human Primary Visual Cortex." Neuropsychologia 83 (March): 161-69.

Murray, R. F., A. B. Sekuler, and P. J. Bennett. 2001. "Time Course of Amodal Completion Revealed by a Shape Discrimination Task." Psychonomic Bulletin \& Review 8 (4): 713-20.

Nanay, Bence. 2010. "Perception and Imagination: Amodal Perception as Mental Imagery." Philosophical Studies 2 (150): 239-54.

Odegaard, Brian, Min Yu Chang, Hakwan Lau, and Sing-Hang Cheung. 2018. "Inflation versus Filling-in: Why We Feel We See More than We Actually Do in Peripheral Vision." Philosophical Transactions of the Royal Society of London. Series B, Biological Sciences 373 (1755). https://doi.org/10.1098/rstb.2017.0345.

Odegaard, Brian, and Hakwan Lau. 2016. "Methodological Considerations to Strengthen Studies of Peripheral Vision.” Trends in Cognitive Sciences, July. https://doi.org/10.1016/j.tics.2016.06.005.

Pajani, Auréliane, Peter Kok, Sid Kouider, and Floris P. de Lange. 2015. "Spontaneous Activity Patterns in Primary Visual Cortex Predispose to Visual Hallucinations.” Journal of Neuroscience 35 (37): 12947-53.

Phillips, Ian. 2011. "Perception and Iconic Memory: What Sperling Doesn't Show.” Mind \& Language 26 (4): $381-411$.

- 2018. "The Methodological Puzzle of Phenomenal Consciousness." Philosophical Transactions of the Royal Society of London. Series B, Biological Sciences 373 (1755): 20170347.

Pylyshyn, Z. 1999. "Is Vision Continuous with Cognition? The Case for Cognitive Impenetrability of Visual Perception." The Behavioral and Brain Sciences 22 (3): 341-65; discussion 366-423.

Rahnev, Dobromir, Brian Maniscalco, Tashina Graves, Elliott Huang, Floris P. de Lange, and Hakwan Lau. 2011. “Attention Induces Conservative Subjective Biases in Visual Perception." Nature Neuroscience 14 (12): 1513-15.

Ramsøy, Thomas Zoëga, and Morten Overgaard. 2004. "Introspection and Subliminal Perception." Phenomenology and the Cognitive Sciences 3 (1): 1-23.

Rensink, R. A., and J. T. Enns. 1998. "Early Completion of Occluded Objects." Vision Research 38 (15-16): 2489-2505.

Rosenthal, Orna, Shinsuke Shimojo, and Ladan Shams. 2009. "Sound-Induced Flash Illusion Is Resistant to Feedback Training." Brain Topography 21 (3-4): 185-92.

Samaha, Jason, and Rachel Denison. 2020. "The Positive Evidence Bias in Perceptual Confidence Is Not Post-Decisional.” bioRxiv. https://doi.org/10.1101/2020.03.15.991513.

Samaha, Jason, Luca Iemi, Saskia Haegens, and Niko A. Busch. 2020. "Spontaneous Brain Oscillations and Perceptual Decision-Making." Trends in Cognitive Sciences 24 (8): 639-53.

Samaha, Jason, Luca Iemi, and Bradley R. Postle. 2017. "Prestimulus Alpha-Band Power Biases Visual Discrimination Confidence, but Not Accuracy." Consciousness and Cognition 54 (September): 47-55.

Samaha, Jason, Joshua J. LaRocque, and Bradley R. Postle. 2020. "Spontaneous Alpha-Band Amplitude Predicts Subjective Visibility but Not Discrimination Accuracy during High-Level Perception." bioRxiv. https://doi.org/10.1101/2020.07.13.201178. 
Sekuler, A. B., and S. E. Palmer. 1992. "Perception of Partly Occluded Objects: A Microgenetic Analysis." Journal of Experimental Psychology. General 121 (1): 95-111.

Shams, L., Y. Kamitani, and S. Shimojo. 2000. "Illusions. What You See Is What You Hear.” Nature 408 (6814): 788.

Solovey, Guillermo, Guy Gerard Graney, and Hakwan Lau. 2015. “A Decisional Account of Subjective Inflation of Visual Perception at the Periphery." Attention, Perception \& Psychophysics 77 (1): 258-71.

Stocker, Alan A., and Eero P. Simoncelli. 2006. "A Model of Self-Consistent Perception." S. Baldassi, N. Megna, D.C. Burr. Visual Clutter Causes High-Magnitude Errors. PLoS 4 (3): e56.

Thielen, Jordy, Sander E. Bosch, Tessa M. van Leeuwen, Marcel A. J. van Gerven, and Rob van Lier. 2019. "Neuroimaging Findings on Amodal Completion: A Review." I-Perception 10 (2): 2041669519840047.

Ward, Emily J. 2018. "Downgraded Phenomenology: How Conscious Overflow Lost Its Richness." Philosophical Transactions of the Royal Society of London. Series B, Biological Sciences 373 (1755). https://doi.org/10.1098/rstb.2017.0355.

Ward, Emily J., Adam Bear, and Brian J. Scholl. 2016. "Can You Perceive Ensembles without Perceiving Individuals?: The Role of Statistical Perception in Determining Whether Awareness Overflows Access." Cognition 152: 78-86.

Webb, Taylor W., Kajsa M. Igelström, Aaron Schurger, and Michael S. A. Graziano. 2016. "Cortical Networks Involved in Visual Awareness Independent of Visual Attention." Proceedings of the National Academy of Sciences 113 (48): 13923-28.

Weisstein, Naomi, Fanny Montalvo, and Gregory Ozog. 1972. "Differential Adaptation to Gratings Blocked by Cubes and Gratings Blocked by Hexagons: A Test of the Neural Symbolic Activity Hypothesis." Psychonomic Science 27 (2): 89-91.

Witt, Jessica K., J. Eric T. Taylor, Mila Sugovic, and John T. Wixted. 2015. "Signal Detection Measures Cannot Distinguish Perceptual Biases from Response Biases.” Perception 44 (3): 289-300. 